\title{
From Cyclic Nanorings to Single-Walled Carbon Nanotubes: Disclosing the Evolution of their Electronic Structure with the Help of Theoretical Methods
}

A. Pérez-Guardiola ${ }^{a}$, R. Ortiz-Cano ${ }^{a, b, c}$, M. E. Sandoval-Salinas ${ }^{d, e}$, J. Fernández-Rossier ${ }^{b, c}$, D. Casanova ${ }^{e, f}$,

A. J. Pérez-Jiménez ${ }^{a}$, and J. C. Sancho-García ${ }^{a *}$

${ }^{a}$ Department of Physical Chemistry,

University of Alicante,

E-03080 Alicante, Spain

${ }^{b}$ Department of Applied Physics,

University of Alicante,

E-03080 Alicante, Spain

${ }^{c}$ QuantaLab,

International Iberian Nanotechnology Laboratory (INL), 4715-330 Braga, Portugal

${ }^{d}$ Departament de Ciéncia de Materials i Química Física, Institut de Química Teòrica i Computacional (IQTCUB),

Universitat de Barcelona,

E-08028 Barcelona, Spain

${ }^{e}$ Donostia International Physics Center (DIPC),

E-20018 Donostia, Spain

${ }^{f}$ IKERBASQUE,

Basque Foundation for Science,

E-48013 Bilbao, Spain

October 19, 2018 
*E-mail: jc.sancho@ua.es 


\begin{abstract}
We systematically investigate the relationships between structural and electronic effects of finite size zigzag or armchair carbon nanotubes of various diameters and lengths, starting from a molecular template of varying shape and diameter, i.e. cyclic oligoacene or oligophenacene molecules, and disclosing how adding layers and/or end-caps (i.e. hemifullerenes) can modify their (poly)radicaloid nature. We mostly used tight-binding and finite-temperature density-based methods, the former providing a simple but intuitive picture about their electronic structure, and the latter dealing effectively with strong correlation effects by relying on a fractional occupation number weighted electron density $\left(\rho^{\mathrm{FOD}}\right)$, with additional RAS-SF calculations backing up the latter results. We also explore how minor structural modifications of nanotube end-caps might influence the results, showing that topology, together with the chemical nature of the systems, is pivotal for the understanding of the electronic properties of these and other related systems.
\end{abstract}

Key words: Organic nanorings, cyclacenes, cyclophenacenes, SWCNT, end-capping, fractional orbital occupation, FT-DFT, RAS-SF. 


\section{Introduction}

Over the past decades, there has been a growing interest for studying open-shell electronic structures of Polycyclic Aromatic Hydrocarbons (PAH). These polyradicaloid molecules, often with planar conjugated backbones, were synthetically elusive until very recently when phenalenyl, or in general larger triangulene-like derivatives, were experimentally obtained with great promise for technological applications. ${ }^{1-4}$ All these molecules are traditionally categorized as open-shell non-Kekulé systems, containing unpaired electrons due to topological effects. However, further extension to polyradical backbones such as zethrenes, ${ }^{5}$ or in general to nanographene radicals, ${ }^{6}$ has recently shown particularly interesting singlet-fission ${ }^{7}$ or nonlinear optics capabilities, ${ }^{8}$ to name just a few of envisioned applications to be exploited in photophysics or excitonic-based fields. The ground state spin multiplicity of alternating hydrocarbons can be predicted by means of the Ovchinnikov's rule, later generalized by the Lieb's theorem, ${ }^{9}$ for which the spin quantum number of a system is given by the expression $S=\frac{\left|N_{A}-N_{B}\right|}{2}$, with $N_{A}\left(N_{B}\right)$ the number of $\mathrm{C}$ atoms in a bipartite lattice. Consequently, the energetical (near-)degeneracy of their one-electron electronic states, also known as zero-energy modes, is given by the sublattice imbalance $N_{Z}=N_{A}-N_{B}$. On the other hand, there is a large variety of PAHs with a singlet ground-state but nevertheless a significant bi- or poly-radical character, with long acenes being probably the paradigmatic example. The latter systems are known to exhibit an increasing poly-radical character with the number of fused benzene rings, with systems longer than pentacene experimentally reported not so far ago and not without significant synthetic efforts. ${ }^{10-13}$ 
This well-established scenario has been altered after the recent emergence of cyclic nanorings and nanobelts, i.e. cyclic organic molecules of limited size and specific edges, constituted by fused and tilted benzene rings ${ }^{14-21}$ until closing the loop. Note that these molecules represent the shortest segment of armchair or zigzag Single-Walled Carbon NanoTubes (SWCNT) and are thus envisioned as molecular templates for the growth of SWCNT of controlled diameter and shape. Whereas CycloParaPhenylene (CPP) compounds are synthesized in a variety of experimental conditions and yields, CyclaCenes (CC) are very elusive so far ${ }^{22}$ with their reactivity possibly related to their polyradical (di- and tetra- depending on the even or odd number of rings, $n$, respectively) character as it was previously argued ${ }^{23-25}$ and recently disclosed by more sophisticated state-of-the-art theoretical methods. ${ }^{26}$ As an intermediate case, we find CycloPHenacene (CPH) compounds, for which an isomer of [12] CPH ([3]cyclobenzo[a]anthracene) has been successfully synthesized very recently. ${ }^{27}$ Figure 1 shows the chemical structure of $[n] \mathrm{CC}$ and $[n] \mathrm{CPH}$, with $n$ indicating the number of fused rings forming the cyclic structures. However, how and why the electronic structure of these finite-size nanorings might change with their structural features (i.e. diameter $-n-$, form of the edges, length $-L-$, and caps) towards the step-bystep formation of SWCNT, is still a matter of investigation that we will systematically tackle here.

Therefore, taking necessarily into account the expected open-shell electronic structure of these cyclic systems, the choice of the theoretical method is far from being trivial. Multi-Configurational (MC) methods have been used in the past for open-shell systems with great success, ${ }^{28}$ but their pronounced computational scaling with the system size precludes its applica- 
tion to linear, planar, or cyclic PAHs composed of more than a few tens of carbon atoms. On the other hand, the cost-effective Density-Functional Theory (DFT) has been traditionally discarded for these (partly) open-shell systems because of the spin-contamination problem and/or the historical difficulties to deal with (near-)degeneracy effects, ${ }^{29,30}$ noting some recent efforts to model the electronic structure of open-shell singlet biradicals by time-dependent versions. ${ }^{31}$ Hence, another way to afford these challenging effects is by relying on a fractional occupation formalism, mimicking thus the occupancy of the open-shell configurations and preventing spin contamination issues at a reasonable computational cost. The fractional occupation is obtained by imposing a Fermi-Dirac (i.e. a Fermi-smearing) distribution under the effect of a fictitious temperature to force that occupation, often dubbed this technique as Finite-Temperature (FT-)DFT ${ }^{32}$ or Thermally-Assisted-Occupation (TAO-)DFT. ${ }^{33,34}$ This formalism also provides qualitatively right density distributions, and it could thus be applied to systems of any chemical nature and structural topology, particularly interesting for PAHs, ${ }^{35}$ which we will exploit here for pristine or end-capped $[n] \mathrm{CC}$ and $[n] \mathrm{CPH}$ cyclic compounds of various diameters and lengths. We

finally compare these results with those from the Restricted-Active-Space Spin-Flip (RAS-SF) method. ${ }^{36}$

\section{Theoretical framework}

\subsection{The FT-DFT method}

Fractional orbital occupation associated with (near-)degeneracy effects, which in turns arise from static or non-dynamical correlation effects, becomes intrinsically difficult to treat by any standard DFT methodology. 
These fractional occupation numbers $\left(0 \leq f_{i} \leq 1\right)$ are known to affect the electronic density, built from the set of occupied orbitals $\left\{\varphi_{i}\right\}$ selfconsistently obtained, through the following expression:

$$
\rho(\mathbf{r})=\sum_{i}^{\infty} f_{i}\left|\varphi_{i}(\mathbf{r})\right|^{2},
$$

and are determined from a Finite-Temperature (FT) Fermi-Dirac distribution: ${ }^{37}$

$$
f_{i}=\frac{1}{1+e^{\left(\epsilon_{i}-E_{F}\right) / \theta}},
$$

depending critically on the $\epsilon_{i}-E_{F}$ difference, with $\epsilon_{i}$ the eigenenergies of $\varphi_{i}$ and $E_{F}$ the Fermi level, and on $\theta=k_{B} T_{e l}$, with $k_{B}$ the Boltzmann constant and $T_{e l}$ a pseudo-temperature used to self-consistently minimize the Gibbs electronic free energy $\left(G_{e l}=E_{e l}-T_{e l} S_{e l}\right)$ of the system. Once a set of fractionally occupied orbitals is generated following the above procedure, we can define a Fractional Occupation Density (FOD) as a real-space measure of static correlation effects: ${ }^{38}$

$$
\rho^{\mathrm{FOD}}(\mathbf{r})=\sum_{i}\left(\delta_{1}-\delta_{2} f_{i}\right)\left|\varphi_{i}(\mathbf{r})\right|^{2},
$$

with $\delta_{1}$ and $\delta_{2}$ chosen to be $(1,1)$ if the eigenenergy $\left(\epsilon_{i}\right)$ is lower than the energy of the Fermi level, $E_{F}$, or $(0,-1)$ otherwise. This orbital-based representation has shown to display useful information about the distribution of unpaired electrons in molecular systems ${ }^{3,39,40}$ and can be integrated:

$$
\mathrm{N}_{\mathrm{FOD}}=\int \rho^{\mathrm{FOD}}(\mathbf{r}) d \mathbf{r}
$$

to yield the $\mathrm{N}_{\mathrm{FOD}}$ value estimating the number of strongly correlated electrons of the system. This cost-effective FT-DFT methodology has been applied before to a variety of chemical (bio)systems ${ }^{41}$ and to small-size linear and cyclic oligoacenes, ${ }^{26}$ with the latter results in good agreement with findings from the RAS-SF method, ${ }^{36}$ showing thus its potential to deal with 
larger systems out of the scope of more costly methods.

\subsubsection{Computational details}

The ORCA 4.0.0.2 package $^{42}$ was used for all the FT-DFT calculations reported here. All compounds studied were optimized at the TPSSD3(BJ)/def2-SVP level, i.e. employing a dispersion-corrected functional to efficiently incorporate intra-molecular non-covalent (i.e. dispersion) effects. ${ }^{43}$ The corresponding FOD-based calculations were done at the (unrestricted) TPSS/def2-TZVP level, ${ }^{44}$ and with the default temperature $T_{e l}=$ $5000 \mathrm{~K}$ as recommended for this functional. ${ }^{38,41}$ The use of a hybrid version (i.e. TPSS0 with a $25 \%$ of exact exchange) with a modified temperature $\left(10000 \mathrm{~K}\right.$ according to the relationship $\left.T_{e l} / K=5000+20000 \cdot a_{x}\right)$ was also tested without significantly modifying the results. Singlet, triplet, and charged energies were obtained by imposing the adequate charge and multiplicity, but without modifying the level of theory fixed above. We increased systematically in all cases the thresholds for SCF calculations (i.e. TightSCF) and numerical integration (i.e. Grid6, NoFinalGrid). The iso-

contour values for displaying the $\rho^{\mathrm{FOD}}(\mathbf{r})$ density were consistently set to $0.005 \mathrm{e} / \mathrm{bohr}^{3}$, and the plots were generated with the UCSF Chimera ${ }^{45}$ (version 1.12) package after proper manipulation of output files. The $\mathrm{N}_{\mathrm{FOD}}$ values are directly extracted from the output file of the calculations.

\subsection{The RAS-SF method}

Spin-Flip (SF) methods rely on a excitation operator promoting $\alpha$ electrons into empty $\beta$ orbitals, which together with an adequate choice of an active space of orbitals, the Restricted Active Space (RAS), can deal with de- 
generacies or near-degeneracies of electronic states. ${ }^{46,47}$ Actually, the combination known as RAS-SF has allowed to accurately treat molecular systems with radical or polyradical character. The number of unpaired electrons is quantified through the expression: ${ }^{48}$

$$
\mathrm{N}_{\mathrm{U}}=\sum_{i}\left(1-\operatorname{abs}\left(1-n_{i}\right)\right)
$$

where $n_{i}$ are the electron occupancies of the RAS-SF natural orbitals $(0 \leq$ $n_{i} \leq 2$ ). Note that Eqs. (4) and (5) are equivalent to each other (i.e. $\mathrm{N}_{\mathrm{FOD}} \equiv \mathrm{N}_{\mathrm{U}}$ ) and actually the RAS-SF calculated fractionally occupied orbitals can be also used to define the corresponding fractional occupation density.

\subsubsection{Computational details}

The RAS-SF calculations were performed with the Q-Chem program ${ }^{49}$ using the lowest ROHF (Restricted Open-Shell) triplet state as a reference configuration in all the systems. The (restricted) active space consisted on all virtuals and doubly occupied $\pi$-orbitals for the RAS1 and RAS3 subspaces, respectively, with 8 electrons in $8 \pi$-orbitals for the RAS2 subspace. We disregard excitations from core electrons (1s C orbitals) and to virtual orbitals with energies higher than 0.5 a.u. for computational efficiency, and use the cost-effective $6-31 \mathrm{G}(\mathrm{d})$ basis set herein.

\section{Results and discussion}

\subsection{Physics provided by the tight-binding method}

We first studied Cyclacenes under the Tight-Binding (TB) approximation. This permits to connect with previous work about the electronic struc- 
tures of short-sized CNTs, ${ }^{50,51}$ and it provides a simple yet intuitive picture of what one would expect next employing more sophisticated theoretical methods. We consider a tight-binding model with one $\pi$ orbital per carbon atom, with first neighbour hopping $t$. The single-particle energy levels computed with this model for cyclic polyenes are shown in Figure 2 for four structures with different values of $L$ and $n$. We remark the following results: (i) For even values of $n$, the eigenvalue spectra has two zero-energy modes, with each one strongly localized at one of the zigzag edges regardless of values of $L$; and (ii) when $n$ is odd and $L=1$, there are no strict zero-energy modes, but two energy split doublets. We have verified that the the splitting decreases dramatically with $L$ (see Figure 2).

The existence of both zero energy states and "quasi" zero energy states can be rationalized as follows. Structures with $L=1$ can be thought as 2 rings with $2 n$ carbon atoms each. The inter-ring coupling occurs only between sites with the same parity, either odd or even, and fortunately the tight-binding problem of a single ring can be solved analytically. For a single ring with $N=2 * n$ sites, the amplitude of the single-particle state on the site $\ell$ of the ring is $\psi_{k}(\ell)=e^{i k \ell}$. The eigenvalue associated with such $\psi_{k}$ is $\epsilon(k)=2 t \cos k=2 t \cos \left(\frac{2 \pi m}{N}\right)$. The permitted values of $k$ are obtained by imposing that the amplitude satisfies $\psi_{k}(\ell)=\psi_{k}(\ell+N)$ from which we infer $k=\frac{2 m \pi}{N}$ where $0, \pm 1, \pm 2 \ldots$ The number of states is thus given by $N$. If we now look for zero energy states, $\epsilon(k)=0$, this happens when $\left(\frac{2 \pi m}{N}\right)=\frac{ \pm \pi}{2}$, or $m=N / 4$. Therefore, for even $n$ values, $N=2 n$ is divisible by 4 and there are two zero modes in the ring. If $n$ is odd, $2 N$ is not divisible by 4 , and there are not zero modes in the ring. 
We now discuss how to build the zero modes of the $L=1$ structures, based on the zero modes of the even $n$ rings. For that matter, we realize that the wave functions $\psi_{z}(\ell)=\frac{1}{\sqrt{N}} e^{ \pm i \frac{\pi}{2} \ell}$ can now be combined leading to symmetric and antisymmetric states, giving two orthogonal $E=0$ states living in the odd and even sites of the ring, respectively. When the two rings are coupled to form the $L=1$ structure with even $n$, the zero modes hosted in the sites that are not affected by the inter-ring coupling are not perturbed, keeping $E=0$. In contrast, the other pair of zero modes, hosted in sites that are affected by inter-ring interactions, form molecular states with energy $\pm t$ (see Figure 2a).

The previous argument can be extended to larger systems, e.g. $L=3$ formed by fusing two $L=1$ blocks. Before their coupling, each $L=1$ block has 2 zero modes. The coupling affects the carbon atoms where the zero energy modes are hosted, and two zero modes hosted are consequently hybridized, but two zero modes hosted at the top and bottom edge of the structure survive. Therefore, regardless the value of $L$, the structure has two sublattice polarized $E=0$ zero modes (see Figure S1 in the Supporting Information). This is a non-trivial result, since the existence of $E=0$ modes is secured when there are more atoms in one of the two triangular sublattices, which is not the case of these systems. The ultimate origin of these zero modes relies on the existence of a structural symmetry that commutes with the sublattice operator. ${ }^{52}$ Therefore, local distortions would lift the degeneracy of the zero mode doublet.

We now consider the case of odd- $n$ structures, for which the elementary rings do not have zero energy modes. Instead, they have 2 doublets 
that overshoot/undershoot the $k= \pm \frac{\pi}{2}$ condition, with energy close to, but strictly different from zero. When fused into the $L=1$ structure, these states give also 4 low energy modes. However, as $L$ increases, keeping fixed $n$, the energy of these 4 states gets closer to $E=0$. We can understand this starting from the limit of a infinite graphene ribbon with zigzag edges and width $L$. It is well known ${ }^{53}$ that the resulting energy bands have two quasi-flat bands with energy close to $E=0$, that vanish exactly at $k= \pm \pi a$, where $a$ is the unit cell length. The energy levels of our finite size nanotubes can be considered as a sampling in the $k$ space of the spectrum of these ribbons. For even- $n$ structures, the sampling is such that it contains the $k= \pm \frac{\pi}{a}$ doublet. For odd- $n$ structures, the sampling is such that it misses that point, leading to a quartet formed by the two energy bands and the two $k$ points closest to the zone boundary. Note that the latter four states can be linearly combined to obtain four sublattice polarized zero-energy modes; two at one zigzag edge, and two at the other edge (see for instance our recent work ${ }^{54}$ ). Increasing $n$ and $L$ will result in a larger number of quasi zero energy modes. The case of $[n] \mathrm{CPH}$ was also analyzed, see Figure S2 in the Supporting Information, without displaying any zero-energy modes in this case. These results from the tight-binding model qualitatively agree with the diradical and tetraradical character of even and odd ciclacenes, respectively, disclosed previously and confirmed quantitatively by previous multiconfigurational wavefunction calculations by some of the authors. ${ }^{26}$

\subsection{Energy magnitudes for increasingly longer nanorings}

As key energy magnitudes for understanding the underlying electronic structure of these systems, we choose the Singlet-Triplet energy difference $\left(\Delta E_{\mathrm{ST}}\right)$, the Vertical Ionization Potential (VIP), the Vertical Electron Affin- 
ity (VEA), and the Quasi-Energy Gap (QEG), defined respectively as:

$$
\begin{aligned}
\Delta E_{\mathrm{ST}} & ={ }^{3} E_{N}-{ }^{1} E_{N}, \\
\mathrm{VIP} & ={ }^{2} E_{N-1}-{ }^{1} E_{N}, \\
\mathrm{VEA} & ={ }^{1} E_{N}-{ }^{2} E_{N+1}, \\
\mathrm{QEG} & =\left({ }^{2} E_{N+1}-{ }^{2} E_{N-1}\right)-{ }^{1} E_{N},
\end{aligned}
$$

with ${ }^{S} E_{N}$ being the FT-DFT calculated total energy of the $N$-electron system with spin multiplicity $S$, and $N+1 / N-1$ indicating the corresponding charged systems. The VIP and VEA energies, or simply $I$ and $A$ in the following for simplicity, relate with the chemical potential $(\mu)$ and other indicators to charge donation and acceptance: ${ }^{55}$

$$
\begin{aligned}
\mu & =-\frac{I+A}{2}, \\
\omega & =\frac{(I+A)^{2}}{4(I-A)}, \\
\omega^{-} & =\frac{(3 I+A)^{2}}{16(I-A)}, \\
\omega^{+} & =\frac{(I+3 A)^{2}}{16(I-A)},
\end{aligned}
$$

with $\omega$ the electrophilicity, and $\omega^{-}\left(\omega^{+}\right)$the electrodonating (electroaccepting) power. Table 1 gathers the results for all the systems considered, with the main findings summarized as follows:

- The $\mathrm{N}_{\mathrm{FOD}}$ values increase with the nanotube length $(L)$ and diameter $(n)$, reaching significantly high values for the longest oligomer of $[n] \mathrm{CC}$, and tend to saturate with values of $L$, with slight differences between odd and even nanotubes. These values indicate their polyradical nature and the growing multiconfigurational character of their singlet ground state. Comparing [12] CC and [12] CPH of the same length, we 
can observe how the values for the latter are roughly halved with respect to the former systems, which agrees with the higher stability of the latter arising from the larger number of aromatic rings according to Clar's rule. ${ }^{56}$

- The $\Delta E_{\mathrm{ST}}$ values decrease with the nanotube length $(L)$ and diameter $(n)$, reaching values as low as $0.1-0.2 \mathrm{eV}$ unless for the case of [12] CPH oligomers. The results of Figure 3 reveal a clear correlation between the singlet-triplet energy difference and the $\mathrm{N}_{\mathrm{FOD}}$ values, a trend previously disclosed for linear and cyclic oligoacenes. ${ }^{26}$ Note how this relationships also qualitatively prevails when RAS-SF/6-31G(d) calculated $\Delta E_{\mathrm{ST}}$ and $\mathrm{N}_{\mathrm{FOD}}$ values are instead considered, for the [12]CC oligomers taken as example (see Figure S2).

- The VIP (VEA) values slightly decrease (increase) with the nanotube length $(L)$, roughly comprising between $5-6(2-3) \mathrm{eV}$ and monotonically converging with the system size, as it was also previously observed for cyclic oligoacenes. ${ }^{57}$ The behaviour of the QEG values, or fundamental gap, is reminiscent of those trends found for VIP and VEA, with their evolution shown in Figure 4. Note that values are expected to converge towards a limit found between $2.0-2.5 \mathrm{eV}$, which is considerably lower than that found for other hoop-shaped conjugated molecules. ${ }^{58}$

- The reactivity indexes considered are related to the maximum electron flow when a molecule is embedded into a bath at a constant potential $v(\mathbf{r})$, and complement the information provided by VIP and VEA for those cases where approximately more or less than one electron can be transferred. ${ }^{59}$ Actually, the values for all systems increase with the nanotube length $(L)$, and follow the sequence $\omega^{-}>\omega>\omega^{+}$inde- 
pendently of $L$ and $n$, thus indicating a propensity to donate rather than to accept charge. The ratio $\frac{\omega^{-}}{\omega^{+}} \simeq 2-3$, with the highest (lowest) ratio found for $[6] \mathrm{CC}([12] \mathrm{CC})$ with $[9] \mathrm{CC}$ and $[12] \mathrm{CPH}$ keeping intermediate values.

This set of results also relates with the energetic stability of finite size tubes, which should ideally correlate with experimental abundance of SWCNT. Fixing the value of $L=6$ for [6] CC, [9] CC, [12] CC, and [12] CPH, which also allows a qualitative comparison with previous theoretical estimates for all the combinations $n+n=8-18$ for both $(n, 0)$ and $(n, n)$ cases, ${ }^{60}$ one can see how the FOD-based descriptors (i.e. $\mathrm{N}_{\mathrm{FOD}}$ ) and energy magnitudes (i.e. $\left.\Delta E_{\mathrm{ST}}\right)$ would predict the following stability: [12] CPH $>[6] \mathrm{CC}>[9] \mathrm{CC}>$ $[12] \mathrm{CC}$, in agreement with the preference for armchair and near-armchair abundance of fragment of SWCNT in the initial steps of their growth. ${ }^{61}$

\subsection{Topology of the FOD density and occupation numbers for increasingly longer nanorings}

We represent in Figure 5 the spatial distribution of $\rho^{\mathrm{FOD}}(\mathbf{r})$, as obtained from FT-DFT calculations, for the shortest $(L=1)$ and longest $(L=6)$ oligomer of all systems considered so far: [6]CC, [9]CC, [12]CC, and $[12] \mathrm{CPH}$. Note that when $L \rightarrow \infty,[n] \mathrm{CC}$ and $[n] \mathrm{CPH}$ systems will lead to zigzag $(n, 0)$ and armchair $(n, n)$ SWCNTs, with the oligomers considered here transitioning between nanobelts and nanorings, respectively, and the infinitely extended systems thoroughly studied in the literature. Note also that for computational studies of finite size SWCNT we need to saturate the edges with $\mathrm{H}$ atoms. For the [6]CC oligomers, it is immediately seen how the density mostly locates at the edges and primarily on the non-bridging $\mathrm{C}$ atoms. Going from $L=1$ to $L=6$ (see Figures S3-S6 
in the Supporting Information) extends the distribution of $\rho^{\mathrm{FOD}}(\mathbf{r})$ to every ring and to all $\mathrm{C}$ atoms within them, while keeping the adherence of (or propensity of finding) the density at the edges. When the diameter of the oligomers increases, i.e. going from $[6] \mathrm{CC}$ to $[9] \mathrm{CC}$ or to $[12] \mathrm{CC}$, the density concentrates almost exclusively on the edges, essentially keeping the same pattern as before regarding on which $\mathrm{C}$ atoms is located. However, the set of [12] CPH oligomers behave completely different, and actually one would need a lower cut-off $\left(\sigma=0.002 \mathrm{e} / \mathrm{bohr}^{3}\right)$ to make the $\rho^{\mathrm{FOD}}(\mathbf{r})$ visible, see Figure S7 in the Supporting Information, indicating a weaker polyradicaloid character in agreement with the larger $\Delta E_{\mathrm{ST}}$ values obtained with the FT-DFT method. Interestingly, the spatial distribution of the RAS-SF $\rho^{\mathrm{FOD}}(\mathbf{r})$ density, see Figure S8 for the set of [12]CC oligomers, closely resembles the ones obtained before. To further illustrate the increase of $\mathrm{N}_{\mathrm{FOD}}$ with $L$ for $[n] C C$ systems, we plot the fractional occupation numbers of a sufficiently large window of orbitals in Figure 6 for the representative [9]CC and $[12] \mathrm{CC}$ cases. The Highest Occupied Molecular Orbital (HOMO) and Lowest Unoccupied Molecular Orbital (LUMO) represent the frontier orbitals, the number of fractionally occupied orbitals distribute around them, showing an increase of the (poly-)radical character with the nanotube size.

To investigate if the increase of $\mathrm{N}_{\mathrm{FOD}}$ values with the nanotube length is due to cumulative or intrinsic effects, we represent in Figure 7 the total values divided by the number of electrons of each system: $\frac{\mathrm{N}_{\mathrm{FOD}}(L)}{\mathrm{N}_{\text {electrons }}}$. We can easily see how for both $[n] \mathrm{CC}$ and $[n] \mathrm{CPH}$ systems each $\mathrm{C}$ atom contributes approximately the same to the polyradicaloid nature of the systems, although more pronouncedly in the case of $[n] \mathrm{CCs}$, with a slight trend to decrease (increase) for $[n] \mathrm{CCs}([n] \mathrm{CPHs})$ as a function of the nanotube 
length $L$. In a second step, we renormalize the $\mathrm{N}_{\mathrm{FOD}}$ values by subtracting the values for the shortest oligomer $(L=1)$ to the rest of the values, and dividing by the number of $\mathrm{C}$ atoms $\left(\mathrm{N}_{\mathrm{C}}\right)$, as given by:

$$
\text { normalized } \mathrm{N}_{\mathrm{FOD}}=\frac{\mathrm{N}_{\mathrm{FOD}}(L)-\mathrm{N}_{\mathrm{FOD}}(L=1)}{\mathrm{N}_{\mathrm{C}}},
$$

highlight the importance of edge effects, i.e. how the inner rings contribute to the global $\mathrm{N}_{\mathrm{FOD}}$ values, which we also represent in Figure 7. Interestingly, the edge effects seem to saturate with the system size for $[n] \mathrm{CC}$ systems, confirming that very long nanotube length would not suffer from infinitely higher and higher $\mathrm{N}_{\mathrm{FOD}}$ values. Note also the nearly constant value of the normalized FOD values for the case of $[n] \mathrm{CPH}$ systems, in agreement with the homogeneous distribution of the FOD density along their structure (see Figure S7 in the Supporting Information). This finding also agrees with previous investigations as the length of the $(n, 0)$ and $(n, n)$ cases increases, even causing a switch to occur in the relative stability of zigzag vs. armchair structures. ${ }^{61}$

\subsection{The effect of end-capping of the longest nanorings}

We also investigate the tailored capping of systems such as [6]CC and [12]CC, both with a $L=6$ length as a matter of illustration, to disclose if the nature of the caps modify significantly or not the electronic properties of finite size nanotubes. We also note that the shape of a nanotube cap strongly depend on the base end, that is, whether a pentagon or a hexagon is situated at the cusp which actually determines the nature of the whole cap. ${ }^{62,63}$ Note that finite size end-capped nanotubes have been proposed as nanovehicles to deliver specific targets into cells after crossing their membranes, ${ }^{64}$ and are reported to magnify the field-emission microscopy images, ${ }^{65}$ opening new 
nanotechnological applications.

Figure 8 displays the $\rho^{\mathrm{FOD}}(\mathbf{r})$ distribution and the associated $\mathrm{N}_{\mathrm{FOD}}$ values for the isolated caps matching the zigzag edges of both [6] CC and [12] CC, at the FT-TPSS/def2-TZVP//TPSS-D3(BJ)/def2-SVP level. We remark how the pentagon base induces a highly delocalized FOD density and a larger $\mathrm{N}_{\mathrm{FOD}}$ value than the hexagon base in all cases, in agreement with what one would expect from the pentagon rule establishing that pentagon-pentagon contacts are energetically more costly. ${ }^{66}$ When these caps are covalently bound to one of the edges of the [6]CC and [12]CC finite nanotubes $(L=6)$, the distribution of $\rho^{\mathrm{FOD}}(\mathbf{r})$, see Figure 9, resembles that found before for non-capped tubes with a concentration of the FOD density at the free edge. Note also the geometric deformation experienced for the tube capped with a pentagon base cap, due to the mismatch between the symmetry of both moieties. Interestingly, we note that always $\mathrm{N}_{\mathrm{FOD}}([n] \mathrm{CC} @ \mathrm{CAP})<\mathrm{N}_{\mathrm{FOD}}$ $([n] \mathrm{CC})+\mathrm{N}_{\mathrm{FOD}}(\mathrm{CAP})$, arising from the passivation of edge effects which in turn attenuates the (poli-)radical character of the systems. In other words, the nature of the $[n] \mathrm{CC} @ \mathrm{CAP}$ is determined primarily by the nature of the tube. When two caps are linked to both edges of the finite nanotube, see Figure 10, the attenuation of the (poli-)radical character is specially remarkable for the [12]CC case, $\mathrm{N}_{\mathrm{FOD}}\left([12] \mathrm{CC} @ \mathrm{CAP}_{2}\right)<<\mathrm{N}_{\mathrm{FOD}}([12] \mathrm{CC})+2$. $\mathrm{N}_{\mathrm{FOD}}(\mathrm{CAP})$, and actually $\mathrm{N}_{\mathrm{FOD}}\left([12] \mathrm{CC} @ \mathrm{CAP}_{2}\right)<\mathrm{N}_{\mathrm{FOD}}([12] \mathrm{CC})$ despite the larger number of $\mathrm{C}$ atoms in [12]CC@ $\mathrm{CAP}_{2}$, and with values close to those found for the $[6] \mathrm{CC} @ \mathrm{CAP}_{2}$ case. Note also that $\mathrm{N}_{\mathrm{FOD}}([n] \mathrm{CC} @ \mathrm{CAP})$ values are always lower when the hexagon base is used to build the cap, for all the cases studied. 


\section{Conclusions}

We have theoretically studied the use of cyclic organic molecules as templates for the controlled growth of carbon nanotubes of finite and welldefined sizes and edges, an issue particularly challenging in the case of zigzag SWCNT due to the (poly-)radical nature of the precursors (i.e. cyclic oligoacenes). Upon a layer-by-layer extension of the nanotube size we have demonstrated that zig-zag nanotubes inherit the (poly-)radical character of the constituting moieties, showing a smooth but gradual decrease of the singlet-triplet energy difference and an increase of the number of unpaired electrons as a function of the number of layers, as well as accessing to other chemically important information not easy to obtain by other techniques. Interestingly, the density arising from the fractionally occupied orbitals, independently of the method used for their calculation, shows a strong adherence to the edge in the case of polyradicaloids independently of their system size and diameter. On the other hand, armchair nanotubes do not show such a pronounced (poly-)radical character since the constituting unit (i.e. cyclic phenacenes) already had an attenuated (poly-)radical character, revealing how the topology of the molecular template is pivotal for the electronic structure of the whole nanotube, and actually delocalizing the density arising from the fractionally occupied orbitals along all the structure. We have also investigated the effect of capping the zig-zag finite-size nanotube with tailored caps, thus inducing a weak passivation of the (poly-)radical nature disclosed before. Overall, our calculations show the intricate and delicate interplay between structural and electronic effects in carbon-based nanoforms, particularly challenging in the case of polyradicaloid molecules. 


\section{Acknowledgements}

A.J.P.J. and J.C.S.G acknowledge the project CTQ2014-55073-P from Spanish Government (MINECO/FEDER) and the project AICO/2018/175 from the Regional Government (GVA/FSE). J.F.R. acknowledges the projects MAT2016-78625 from Spanish Government (MINECO/FEDER) and projects No. PTDC/FIS-NAN/4662/2014 and No. PTDC/FIS-NAN/3668/2014 from Portuguese Government (Fundação para a Ciência e Tecnologia). D.C. is thankful to projects IT588-13 (Eusko Jaurlaritza) and CTQ2016-80955 from the Spanish Government (MINECO/FEDER). M.E.S.-S. acknowledges CONACyT-México for a Ph.D. fellowship (ref. 591700). R.O.C. acknowledges "Generalitat Valenciana" and "Fondo Social Europeo" for a Ph.D. fellowship (ACIF/2018/198).

\section{Associated content}

The Supporting Information contains in this order: (i) Sketch showing the formation of zero-energy modes when even (left) and odd (right) symmetrical and equivalent parts of $[n] \mathrm{CC}$ are bound together; (ii) Singlet-triplet energy gaps and $\mathrm{N}_{\mathrm{FOD}}$ of [12]CC with $L=1-6$ computed at the RAS-SF /6$31 \mathrm{G}(\mathrm{d})$ level; (iii) Chemical structures and plots $\left(\sigma=0.005 \mathrm{e} / \mathrm{bohr}^{3}\right)$ of the FOD density as obtained from the FT-DFT method, for the set of $[n] \mathrm{CC}$ and $[n] \mathrm{CPH}$ compounds $(L=1-6)$; (iv) Chemical structures and plots $\left(\sigma=0.002 \mathrm{e} / \mathrm{bohr}^{3}\right)$ of the FOD density as obtained from the FT-DFT method for the set of $[n] \mathrm{CPH}$ compounds $(L=1-6)$; and (v) Chemical structures and plots $\left(\sigma=0.002 \mathrm{e} / \mathrm{bohr}^{3}\right)$ of the FOD density as obtained from the RAS-SF method, for the set of [12]CC compounds $(L=1-6)$. 


\section{References}

[1] Inoue, J.; Fukui, K.; Kubo, T.; Nakazawa, S.; Sato, K.; Shiomi, D.; Morita, Y.; Yamamoto, K.; Takui, T.; Nakasuji, K. The First Detection of a Clar's Hydrocarbon, 2,6,10-tri-tert-butyltriangulene: A Groundstate Triplet of Non-Kekulé Polynuclear Benzenoid Hydrocarbon. Journal of the American Chemical Society 2001, 123, 12702-12703.

[2] Morita, Y.; Suzuki, S.; Sato, K.; Takui, T. Synthetic Organic Spin Chemistry for Structurally Well-defined Open-shell Graphene Fragments. Nature Chemistry 2011, 3, 197.

[3] Das, A.; Müller, T.; Plasser, F.; Lischka, H. Polyradical Character of Triangular Non-Kekulé structures, Zethrenes, p-QuinodimethaneLinked Bisphenalenyl, and the Clar Goblet in Comparison: An Extended Multireference Study. The Journal of Physical Chemistry A 2016, 120, 1625-1636.

[4] Wang, S.; Talirz, L.; Pignedoli, C. A.; Feng, X.; Müllen, K.; Fasel, R.; Ruffieux, P. Giant Edge State Splitting at Atomically Precise Graphene Zigzag Edges. Nature Communications 2016, 7, 11507.

[5] Lukman, S.; Richter, J. M.; Yang, L.; Hu, P.; Wu, J.; Greenham, N. C.; Musser, A. J. Efficient Singlet Fission and Triplet-pair Emission in a Family of Zethrene Diradicaloids. Journal of the American Chemical Society 2017, 139, 18376-18385.

[6] Desroches, M.; Mayorga Burrezo, P.; Boismenu-Lavoie, J.; Peña Álvarez, M.; Gómez-García, C. J.; Matxain, J. M.; Casanova, D.; Morin, J.-F.; Casado, J. Breaking Bonds and Forming Nanographene Diradicals with Pressure. Angewandte Chemie 2017, 129, 16430-16435. 
[7] López-Carballeira, D.; Casanova, D.; Ruipérez, F. Theoretical Design of Conjugated Diradicaloids as Singlet Fission Sensitizers: Quinones and Methylene Derivatives. Physical Chemistry Chemical Physics 2017, 19, 30227-30238.

[8] Nakano, M.; Champagne, B. Theoretical Design of Open-Shell Singlet Molecular Systems for Nonlinear Optics. The Journal of Physical Chemistry Letters 2015, 6, 3236-3256.

[9] Lieb, E. H. Two Theorems on the Hubbard Model. Physical Review Letters 1989, 62, 1201.

[10] Mondal, R.; Shah, B. K.; Neckers, D. C. Photogeneration of Heptacene in a Polymer Matrix. Journal of the American Chemical Society 2006, 128, 9612-9613.

[11] Tönshoff, C.; Bettinger, H. F. Photogeneration of Octacene and Nonacene. Angewandte Chemie International Edition 2010, 49, 41254128.

[12] Huang, R.; Phan, H.; Herng, T. S.; Hu, P.; Zeng, W.; Dong, S.-q.; Das, S.; Shen, Y.; Ding, J.; Casanova, D.; Wu, J. Higher Order $\pi$ Conjugated Polycyclic Hydrocarbons with Open-Shell Singlet Ground State: Nonazethrene versus Nonacene. Journal of the American Chemical Society 2016, 138, 10323-10330.

[13] Einholz, R.; Fang, T.; Berger, R.; Gruninger, P.; Fruh, A.; Chasse, T.; Fink, R. F.; Bettinger, H. F. Heptacene: Characterization in Solution, in the Solid State, and in Films. Journal of the American Chemical Society 2017, 139, 4435-4442.

[14] Omachi, H.; Segawa, Y.; Itami, K. Synthesis of Cycloparaphenylenes 
and Related Carbon Nanorings: A Step Toward the Controlled Synthesis of Carbon Nanotubes. Accounts of Chemical Research 2012, 45, $1378-1389$.

[15] Segawa, Y.; Fukazawa, A.; Matsuura, S.; Omachi, H.; Yamaguchi, S.; Irle, S.; Itami, K. Combined Experimental and Theoretical Studies on the Photophysical Properties of Cycloparaphenylenes. Organic \& Biomolecular Chemistry 2012, 10, 5979-5984.

[16] Hirst, E. S.; Jasti, R. Bending Benzene: Syntheses of [n]Cycloparaphenylenes. The Journal of Organic Chemistry 2012, 77, 10473-10478.

[17] Yamago, S.; Kayahara, E.; Iwamoto, T. Organoplatinum-Mediated Synthesis of Cyclic $\pi$-Conjugated Molecules: Towards a New Era of ThreeDimensional Aromatic Compounds. The Chemical Record 2014, 14, $84-100$.

[18] Golder, M. R.; Jasti, R. Syntheses of the Smallest Carbon Nanohoops and the Emergence of Unique Physical Phenomena. Accounts of Chemical Research 2015, 48, 557-566.

[19] Kayahara, E.; Patel, V. K.; Xia, J.; Jasti, R.; Yamago, S. Selective and Gram-Scale Synthesis of [6]Cycloparaphenylene. Synlett 2015, 26, $1615-1619$.

[20] Darzi, E. R.; Jasti, R. The Dynamic, Size-Dependent Properties of [5][12]Cycloparaphenylenes. Chemical Society Reviews 2015, 44, 64016410.

[21] Segawa, Y.; Yagi, A.; Matsui, K.; Itami, K. Design and Synthesis of 
Carbon Nanotube Segments. Angewandte Chemie International Edition 2016, 55, 5136-5158.

[22] Lu, X.; Wu, J. After 60 Years of Efforts: The Chemical Synthesis of a Carbon Nanobelt. Chem 2017, 2, 619-620.

[23] Choi, H. S.; Kim, K. S. Structures, Magnetic Properties, and Aromaticity of Cyclacenes. Angewandte Chemie International Edition 1999, 38, $2256-2258$.

[24] Chen, Z.; Jiang, D.-e.; Lu, X.; Bettinger, H. F.; Dai, S.; Schleyer, P. v. R.; Houk, K. N. Open-Shell Singlet Character of Cyclacenes and Short Zigzag Nanotubes. Organic Letters 2007, 9, 5449-5452.

[25] Sadowsky, D.; McNeill, K.; Cramer, C. J. Electronic Structures of [n]Cyclacenes $(n=6-12)$ and Short, Hydrogen-Capped, Carbon Nanotubes. Faraday Discussions 2010, 145, 507-521.

[26] Pérez-Guardiola, A.; Sandoval-Salinas, M. E.; Casanova, D.; SanFabián, E.; Pérez-Jiménez, A.; Sancho-Garcia, J.-C. The Role of Topology in Organic Molecules: Origin and Comparison of the Radical Character in Linear and Cyclic Oligoacenes and Related Oligomers. Physical Chemistry Chemical Physics 2018, 20, 7112-7124.

[27] Povie, G.; Segawa, Y.; Nishihara, T.; Miyauchi, Y.; Itami, K. Synthesis of a Carbon Nanobelt. Science 2017, 356, 172-175.

[28] Szalay, P. G.; Muller, T.; Gidofalvi, G.; Lischka, H.; Shepard, R. Multiconfiguration Self-consistent Field and Multireference Configuration Interaction Methods and Applications. Chemical Reviews 2011, 112, $108-181$. 
[29] Cohen, A. J.; Mori-Sánchez, P.; Yang, W. Challenges for Density Functional Theory. Chemical Reviews 2011, 112, 289-320.

[30] San-Fabián, E.; Moscardó, F. Polarized-Unpolarized Ground State of Small Polycyclic Aromatic Hydrocarbons. International Journal of Quantum Chemistry 2013, 113, 815-819.

[31] Canola, S.; Casado, J.; Negri, F. The Double Exciton State of Conjugated Chromophores with Strong Diradical Character: Insights from TDDFT Calculations. Phys. Chem. Chem. Phys. 2018, 20, 2422724238 .

[32] Grimme, S. Towards First Principles Calculation of Electron Impact Mass Spectra of Molecules. Angewandte Chemie International Edition 2013, 52, 6306-6312.

[33] Chai, J.-D. Density Functional Theory with Fractional Orbital Occupations. The Journal of Chemical Physics 2012, 136, 154104.

[34] Chai, J.-D. Thermally-Assisted-Occupation Density Functional Theory with Generalized-Gradient Approximations. The Journal of Chemical Physics 2014, 140, 18A521.

[35] Yeh, C.-N.; Chai, J.-D. Role of Kekulé and non-Kekulé Structures in the Radical Character of Alternant Polycyclic Aromatic Hydrocarbons: A TAO-DFT Study. Scientific reports 2016, 6, 30562.

[36] Casanova, D.; Head-Gordon, M. Restricted Active Space Spin-Flip Configuration Interaction Approach: Theory, Implementation and Examples. Physical Chemistry Chemical Physics 2009, 11, 9779-9790.

[37] Lin, C.-Y.; Hui, K.; Chung, J.-H.; Chai, J.-D. Self-Consistent Determi- 
nation of the Fictitious Temperature in Thermally-Assisted-Occupation Density Functional Theory. RSC Advances 2017, 7, 50496-50507.

[38] Grimme, S.; Hansen, A. A Practicable Real-Space Measure and Visualization of Static Electron-Correlation Effects. Angewandte Chemie International Edition 2015, 54, 12308-12313.

[39] Plasser, F.; Pašalić, H.; Gerzabek, M. H.; Libisch, F.; Reiter, R.; Burgdörfer, J.; Müller, T.; Shepard, R.; Lischka, H. The Multiradical Character of One-and Two-Dimensional Graphene Nanoribbons. Angewandte Chemie International Edition 2013, 52, 2581-2584.

[40] Horn, S.; Plasser, F.; Müller, T.; Libisch, F.; Burgdörfer, J.; Lischka, H. A Comparison of Singlet and Triplet States for One-and Twodimensional Graphene Nanoribbons Using Multireference Theory. Theoretical Chemistry Accounts 2014, 133, 1511.

[41] Bauer, C. A.; Hansen, A.; Grimme, S. The Fractional Occupation Number Weighted Density as a Versatile Analysis Tool for Molecules with a Complicated Electronic Structure. Chemistry-A European Journal 2017, 23, 6150-6164.

[42] Neese, F. The ORCA Program System. Wiley Interdisciplinary Reviews: Computational Molecular Science 2012, 2, 73-78.

[43] Grimme, S.; Antony, J.; Ehrlich, S.; Krieg, H. A Consistent and Accurate Ab Initio Parametrization of Density Functional Dispersion Correction (DFT-D) for the 94 Elements H-Pu. The Journal of Chemical Physics 2010, 132, 154104.

[44] Tao, J.; Perdew, J. P.; Staroverov, V. N.; Scuseria, G. E. Climbing the Density Functional Ladder: Nonempirical Meta-Generalized Gradient 
Approximation Designed for Molecules and Solids. Physical Review Letters 2003, 91, 146401.

[45] Pettersen, E. F.; Goddard, T. D.; Huang, C. C.; Couch, G. S.; Greenblatt, D. M.; Meng, E. C.; Ferrin, T. E. UCSF Chimera: A Visualization System for Exploratory Research and Analysis. Journal of Computational Chemistry 2004, 25, 1605-1612.

[46] Krylov, A. I. Spin-Flip Configuration Interaction: An Electronic Structure Model that is both Variational and Size-Consistent. Chemical Physics Letters 2001, 350, 522-530.

[47] Krylov, A. I. Spin-flip Equation-of-Motion Coupled-Cluster Electronic Structure Method for a Description of Excited States, Bond Breaking, Diradicals, and Triradicals. Accounts of Chemical Research 2006, 39, $83-91$.

[48] Head-Gordon, M. Characterizing Unpaired Electrons from the OneParticle Density Matrix. Chemical Physics Letters 2003, 372, 508-511.

[49] Shao et al., Y. Advances in Molecular Quantum Chemistry Contained in the Q-Chem 4 Program Package. Molecular Physics 2015, 113, 184215.

[50] Bulusheva, L.; Okotrub, A.; Romanov, D.; Tomanek, D. Electronic Structure of $(n, 0)$ Zigzag Carbon Nanotubes: Cluster and Crystal Approach. The Journal of Physical Chemistry A 1998, 102, 975-981.

[51] Correa, J.; da Silva, A. J.; Pacheco, M. Tight-binding Model for Carbon Nanotubes from ab initio Calculations. Journal of Physics: Condensed Matter 2010, 22, 275503. 
[52] Koshino, M.; Morimoto, T.; Sato, M. Topological Zero Modes and Dirac Points Protected by Spatial Symmetry and Chiral Symmetry. Physical Review B 2014, 90, 115207.

[53] Nakada, K.; Fujita, M.; Dresselhaus, G.; Dresselhaus, M. S. Edge State in Graphene Ribbons: Nanometer Size Effect and Edge Shape Dependence. Physical Review B 1996, 54, 17954-17961.

[54] Ortiz, R.; García-Martínez, N. A.; Lado, J. L.; Fernández-Rossier, J. Electrical Spin Manipulation in Graphene Nanostructures. Physical Review B 2018, 97, 195425.

[55] Gazquez, J. L.; Cedillo, A.; Vela, A. Electrodonating and Electroaccepting Powers. The Journal of Physical Chemistry A 2007, 111, 19661970.

[56] Esser, B. Theoretical Analysis of [5.5.6]Cyclacenes: Electronic Properties, Strain Energies and Substituent Effects. Physical Chemistry Chemical Physics 2015, 17, 7366-7372.

[57] Wu, C.; Lee, P.; Chai, J. Electronic Properties of Cyclacenes from TAODFT. Scientific Reports 2016, 6, 37249.

[58] Liu, J.; Adamska, L.; Doorn, S. K.; Tretiak, S. Singlet and Triplet Excitons and Charged Polarons in Cycloparaphenylenes: A Density Functional Theory Study. Physical Chemistry Chemical Physics 2015, 17, 14613-14622.

[59] Geerlings, P.; De Proft, F.; Langenaeker, W. Conceptual Density Functional Theory. Chemical Reviews 2003, 103, 1793-1874.

[60] Hedman, D.; Barzegar, H. R.; Rosén, A.; Wågberg, T.; Larsson, J. A. 
On the Stability and Abundance of Single Walled Carbon Nanotubes. Scientific reports 2015, 5, 16850.

[61] Hedman, D.; Larsson, J. A. Length Dependent Stability of SingleWalled Carbon Nanotubes and How it Affects their Growth. Carbon 2017, 116, 443-447.

[62] Lair, S.; Herndon, W.; Murr, L.; Quinones, S. End Cap Nucleation of Carbon Nanotubes. Carbon 2006, 44, 447-455.

[63] Melle-Franco, M.; Brinkmann, G.; Zerbetto, F. Modeling Nanotube Caps: The Relationship Between Fullerenes and Caps. The Journal of Physical Chemistry A 2015, 119, 12839-12844.

[64] Höfinger, S.; Melle-Franco, M.; Gallo, T.; Cantelli, A.; Calvaresi, M.; Gomes, J. A.; Zerbetto, F. A Computational Analysis of the Insertion of Carbon Nanotubes into Cellular Membranes. Biomaterials 2011, 32, $7079-7085$.

[65] Tanaka, H.; Akita, S.; Pan, L.; Nakayama, Y. Comparison of Capped Carbon Nanotube with Open-ended One for Field Emission. Japanese Journal of Applied Physics 2004, 43, L427.

[66] Kobayashi, K.; Nagase, S.; Yoshida, M.; Ōsawa, E. Endohedral Metallofullerenes. Are the Isolated Pentagon Rule and Fullerene Structures Always Satisfied? Journal of the American Chemical Society 1997, $119,12693-12694$. 
Table 1: $\mathrm{N}_{\mathrm{FOD}}$ and energy magnitudes (in $\mathrm{eV}$ ) for all systems as a function of their size $(L=1-6)$.

\begin{tabular}{|c|c|c|c|c|c|c|c|c|c|c|}
\hline System & Size & $\mathrm{N}_{\mathrm{FOD}}$ & $\Delta E_{\mathrm{ST}}$ & VIP & VEA & QEG & $\mu$ & $\omega$ & $\omega^{-}$ & $\omega^{+}$ \\
\hline \multirow[t]{6}{*}[6]{$\mathrm{CC}$} & $L=1$ & 1.806 & 0.398 & 5.90 & 1.57 & 4.33 & -2.16 & 3.22 & 5.36 & 1.62 \\
\hline & $L=2$ & 3.259 & 0.227 & 5.75 & 2.04 & 3.71 & -1.85 & 4.09 & 6.27 & 2.37 \\
\hline & $L=3$ & 4.097 & 0.171 & 5.65 & 2.33 & 3.32 & -1.66 & 4.79 & 7.00 & 3.00 \\
\hline & $L=4$ & 4.421 & 0.150 & 5.46 & 2.42 & 3.05 & -1.52 & 5.09 & 7.25 & 3.31 \\
\hline & $L=5$ & 5.081 & 0.134 & 5.42 & 2.60 & 2.82 & -1.41 & 5.69 & 7.17 & 3.87 \\
\hline & $L=6$ & 5.767 & 0.122 & 5.38 & 2.75 & 2.63 & -1.32 & 6.27 & 8.46 & 4.40 \\
\hline \multirow[t]{6}{*}[9]{$\mathrm{CC}$} & $L=1$ & 3.471 & 0.250 & 5.43 & 1.97 & 3.46 & -1.73 & 3.96 & 6.03 & 2.33 \\
\hline & $L=2$ & 4.370 & 0.182 & 5.35 & 2.28 & 3.07 & -1.53 & 4.74 & 6.84 & 3.02 \\
\hline & $L=3$ & 5.027 & 0.149 & 5.27 & 2.48 & 2.79 & -1.40 & 5.39 & 7.50 & 3.62 \\
\hline & $L=4$ & 5.781 & 0.129 & 5.21 & 2.64 & 2.57 & -1.29 & 5.99 & 8.11 & 4.19 \\
\hline & $L=5$ & 6.443 & 0.116 & 5.16 & 2.77 & 2.40 & -1.20 & 6.56 & 8.69 & 4.72 \\
\hline & $L=6$ & 7.036 & 0.107 & 5.12 & 2.87 & 2.25 & -1.13 & 7.09 & 9.23 & 5.24 \\
\hline
\end{tabular}


Table 1 (cont.): $\mathrm{N}_{\mathrm{FOD}}$ and energy magnitudes (in $\mathrm{eV}$ ) for all systems as a function of their size $(L=1-6)$.

\begin{tabular}{|c|c|c|c|c|c|c|c|c|c|c|}
\hline System & Size & $\mathrm{N}_{\mathrm{FOD}}$ & $\Delta E_{\mathrm{ST}}$ & VIP & VEA & QEG & $\mu$ & $\omega$ & $\omega^{-}$ & $\omega^{+}$ \\
\hline \multirow[t]{6}{*}[12]{$\mathrm{CC}$} & $L=1$ & 3.212 & 0.227 & 5.24 & 2.28 & 2.95 & -1.48 & 4.78 & 6.85 & 3.09 \\
\hline & $L=2$ & 5.653 & 0.141 & 5.14 & 2.52 & 2.61 & -1.31 & 5.61 & 7.69 & 3.86 \\
\hline & $L=3$ & 6.766 & 0.116 & 5.08 & 2.69 & 2.40 & -1.20 & 6.30 & 8.39 & 4.51 \\
\hline & $L=4$ & 7.588 & 0.102 & 5.04 & 2.81 & 2.23 & -1.11 & 6.93 & 9.03 & 5.10 \\
\hline & $L=5$ & 8.261 & 0.093 & 5.00 & 2.91 & 2.09 & -1.04 & 7.51 & 9.62 & 5.66 \\
\hline & $L=6$ & 8.863 & 0.087 & 4.97 & 3.00 & 1.97 & -0.98 & 8.08 & 10.2 & 6.21 \\
\hline \multirow[t]{6}{*}[12]{$\mathrm{CPH}$} & $L=1$ & 0.768 & 0.856 & 5.54 & 1.87 & 3.67 & -1.84 & 3.74 & 5.82 & 2.11 \\
\hline & $L=2$ & 1.842 & 0.495 & 5.29 & 2.26 & 3.03 & -1.52 & 4.71 & 6.79 & 3.01 \\
\hline & $L=3$ & 2.475 & 0.363 & 5.16 & 2.48 & 2.69 & -1.34 & 5.44 & 7.52 & 3.69 \\
\hline & $L=4$ & 2.786 & 0.310 & 5.11 & 2.65 & 2.46 & -1.23 & 6.11 & 8.20 & 4.32 \\
\hline & $L=5$ & 4.137 & 0.252 & 5.04 & 2.77 & 2.26 & -1.13 & 6.74 & 8.83 & 4.93 \\
\hline & $L=6$ & 4.183 & 0.221 & 5.00 & 2.89 & 2.11 & -1.06 & 7.36 & 9.46 & 5.52 \\
\hline
\end{tabular}



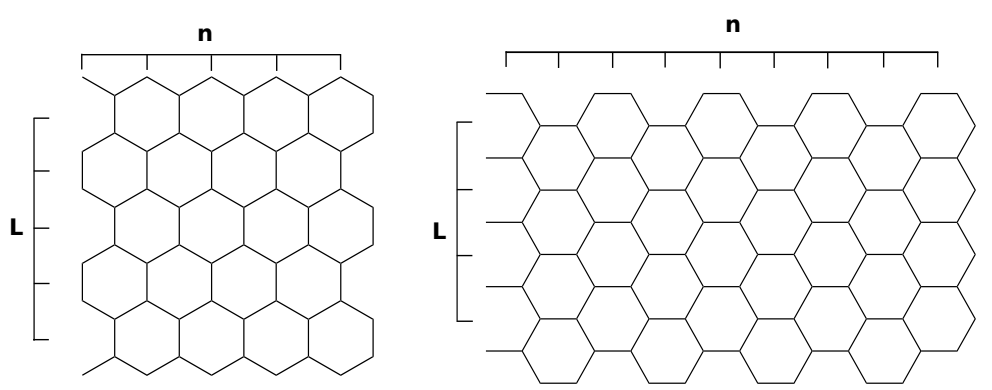

Figure 1: Chemical structures of $[n] \mathrm{CC}$ (left) and $[n] \mathrm{CPH}$ (right) systems, with $\mathrm{H}$ atoms omitted for clarity. The number of fused rings $(n)$ and the length $(L)$ of the corresponding nanotube are also indicated. 


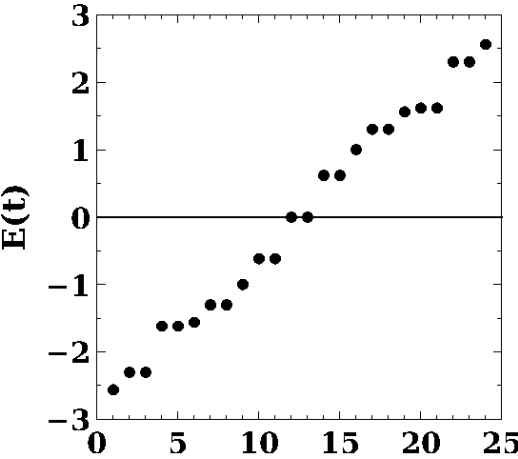

(a)
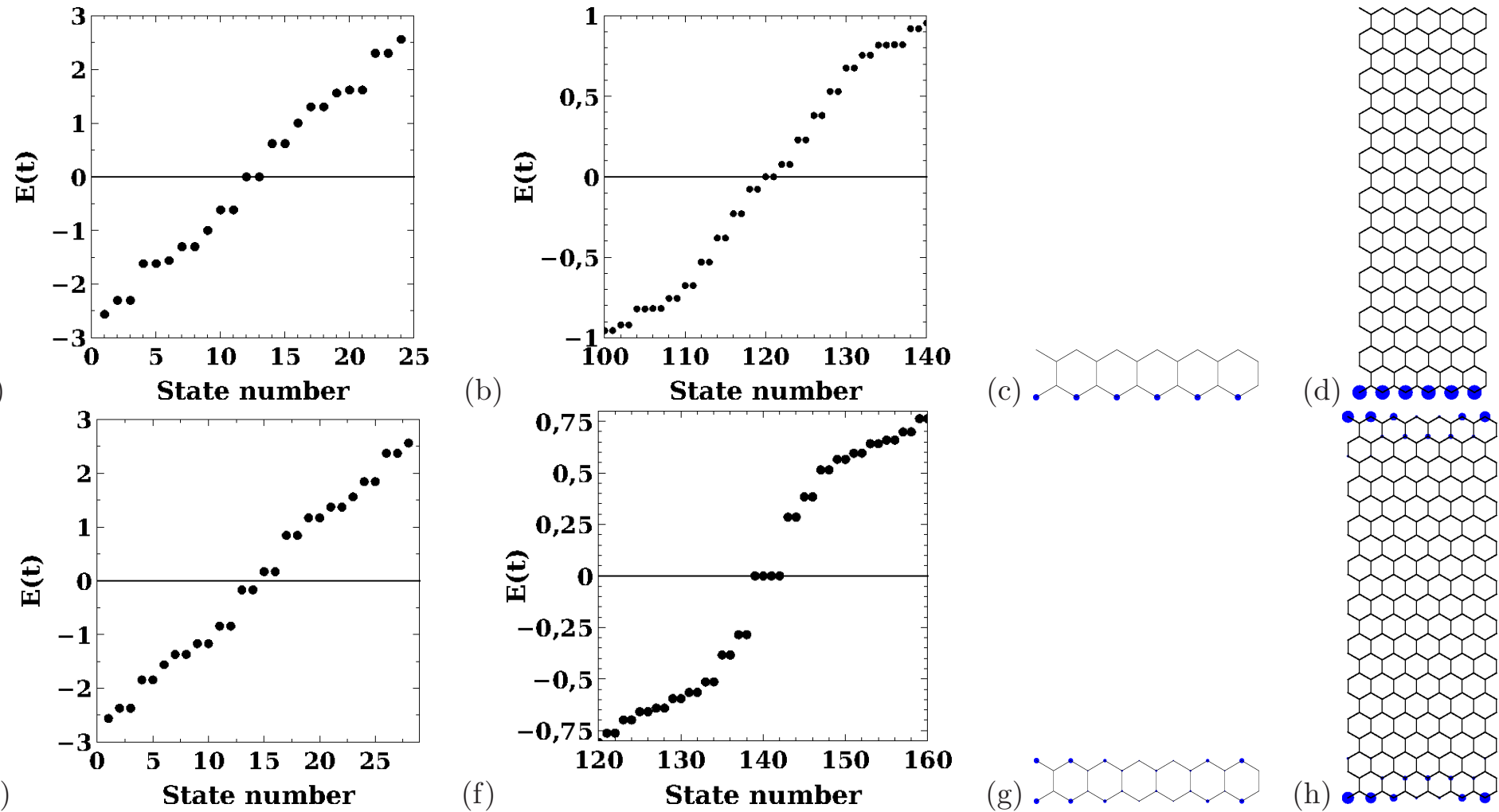

Figure 2: Top: Tight-binding eigenvalue spectra for [6]CC with (a) $L=1$, (b) $L=19$, as well as the plot of one of their corresponding zero-energy modes $-(\mathrm{c})$ and $(\mathrm{d})-$. Bottom: Tight-binding eigenvalue spectra for $[7] \mathrm{CC}$ with $(\mathrm{e}) L=1$, $(\mathrm{f})$ $L=19$, as well as the plot of one of their corresponding ingap states $-(\mathrm{g})$ and $(\mathrm{h})-$. 


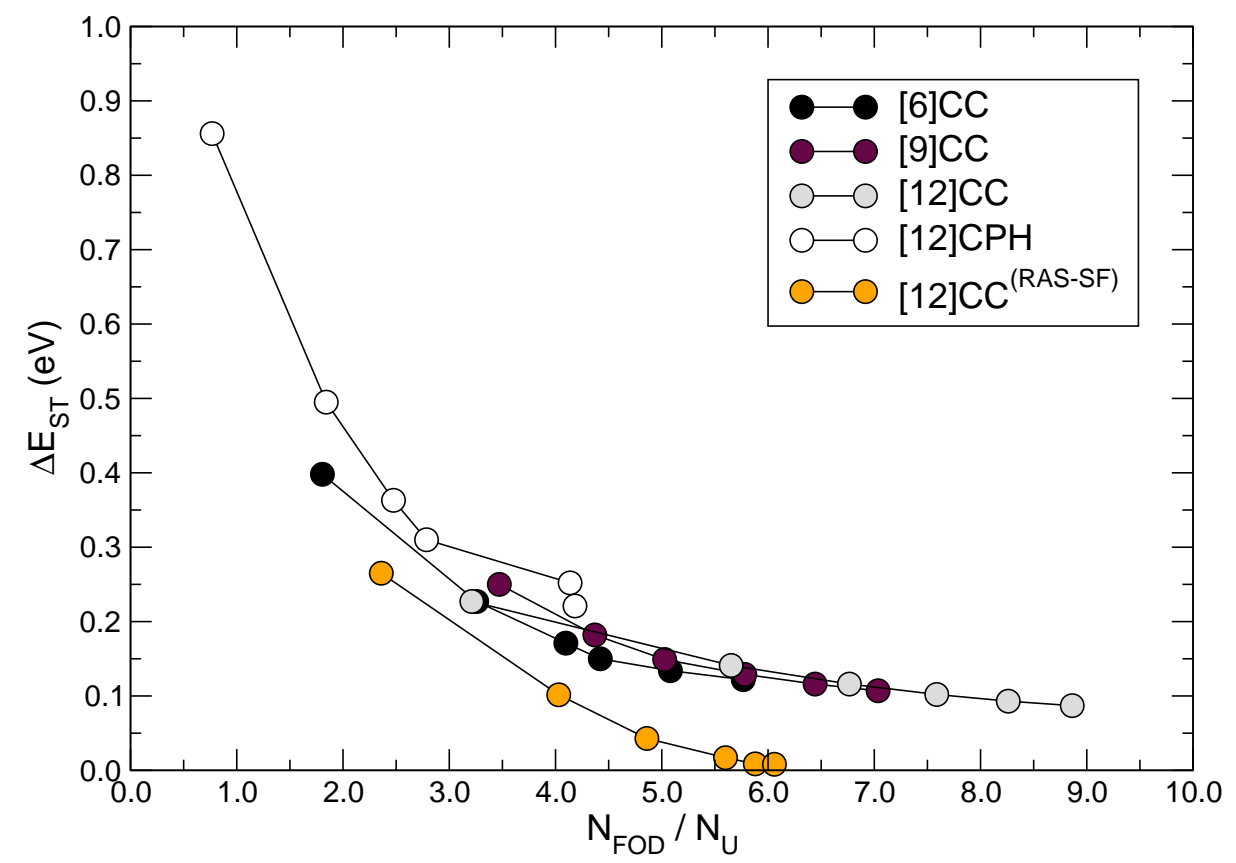

Figure 3: Evolution of the $\Delta E_{\text {ST }}$ values obtained from the FT-DFT method, as a function of the $\mathrm{N}_{\mathrm{FOD}}$ values for for all systems $(L=1-6)$. The $\mathrm{N}_{\mathrm{U}}$ results for $[12] \mathrm{CC}(L=1-6)$ systems are also shown. The straight lines are a guide to the eye. 


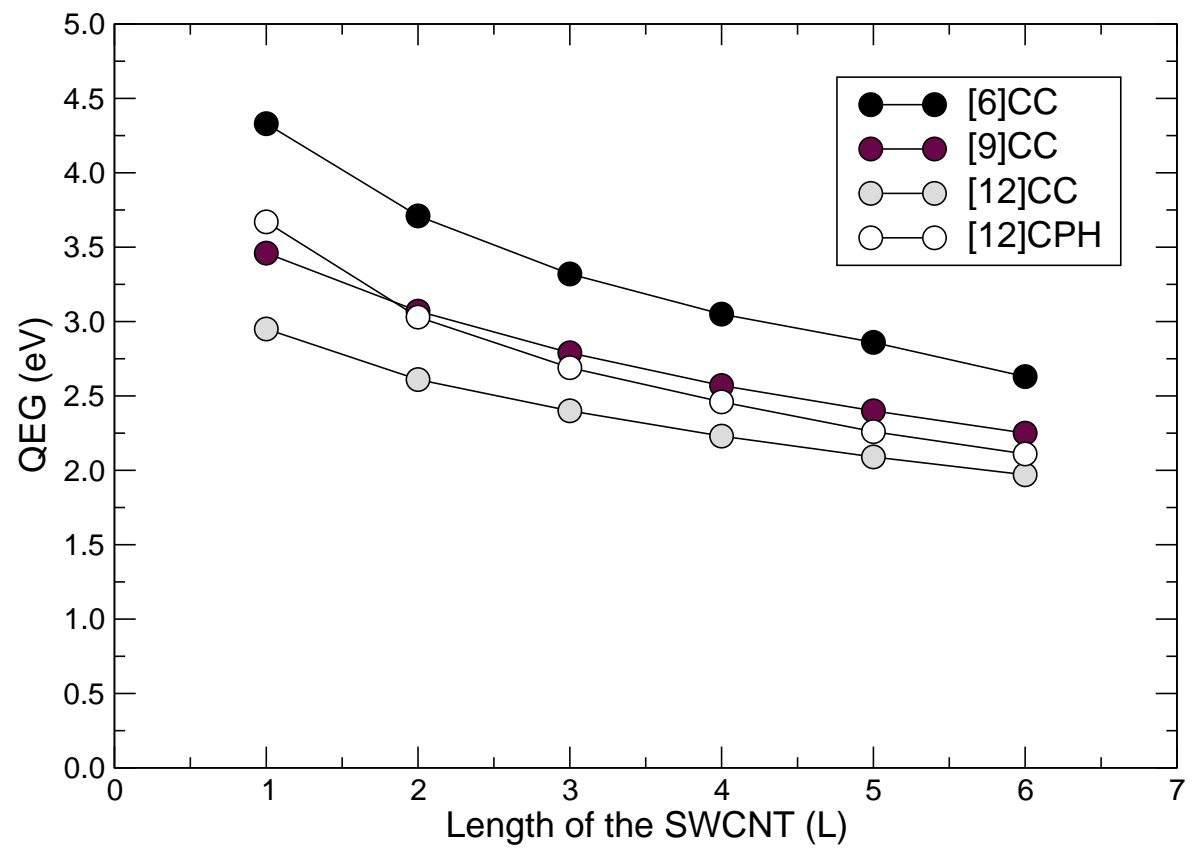

Figure 4: Evolution of the QEG values obtained from the FT-DFT method, as a function of the nanotube size $(L=1-6)$ for all systems. 

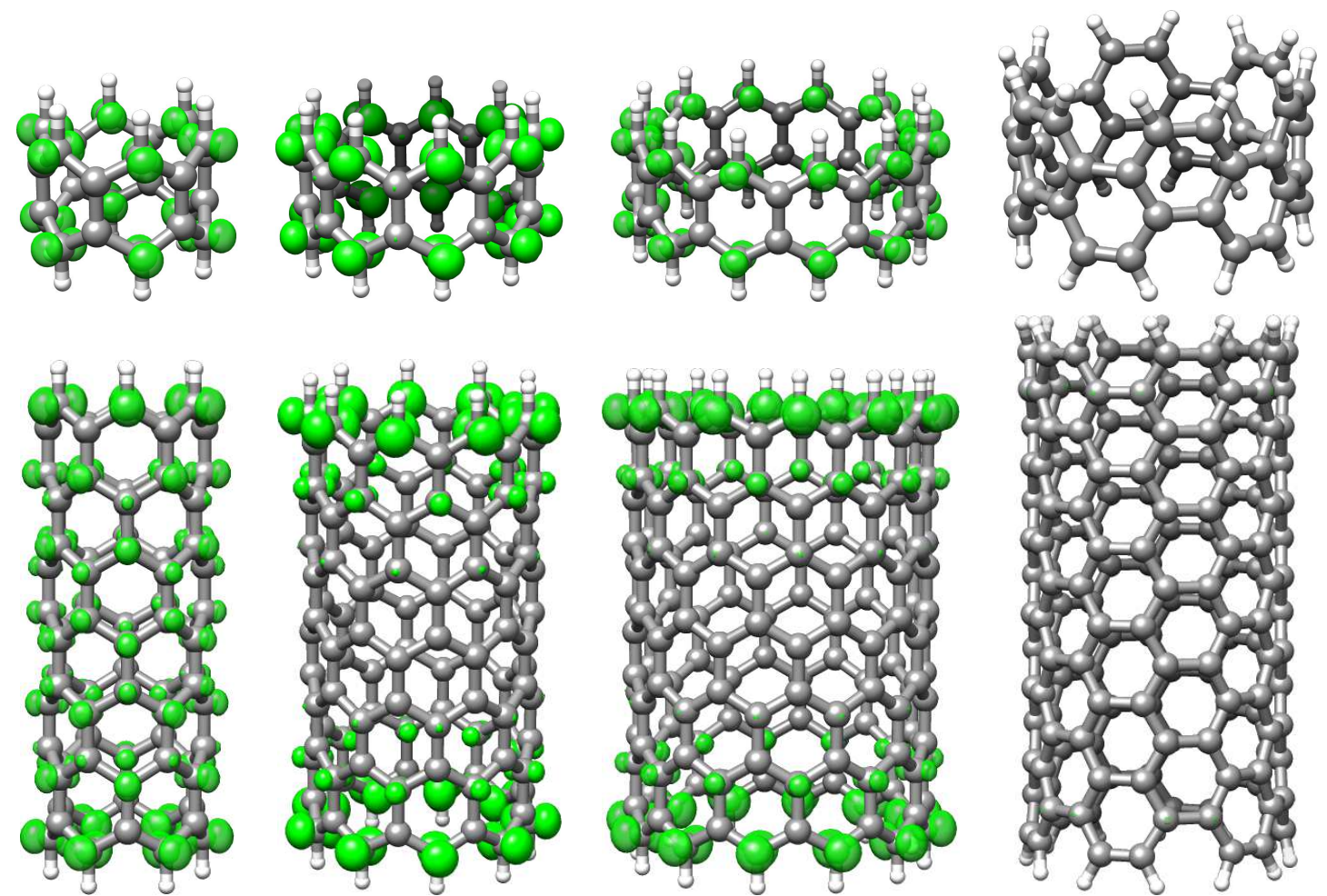

Figure 5: Chemical structures and plots $\left(\sigma=0.005 \mathrm{e} / \mathrm{bohr}^{3}\right)$ of the FOD density obtained from the FT-DFT method, for the shortest (top) and longest (bottom) oligomer of the set of $[n] \mathrm{CC}(n=6,9,12)$ and $[12] \mathrm{CPH}$ compounds (from left to right). 

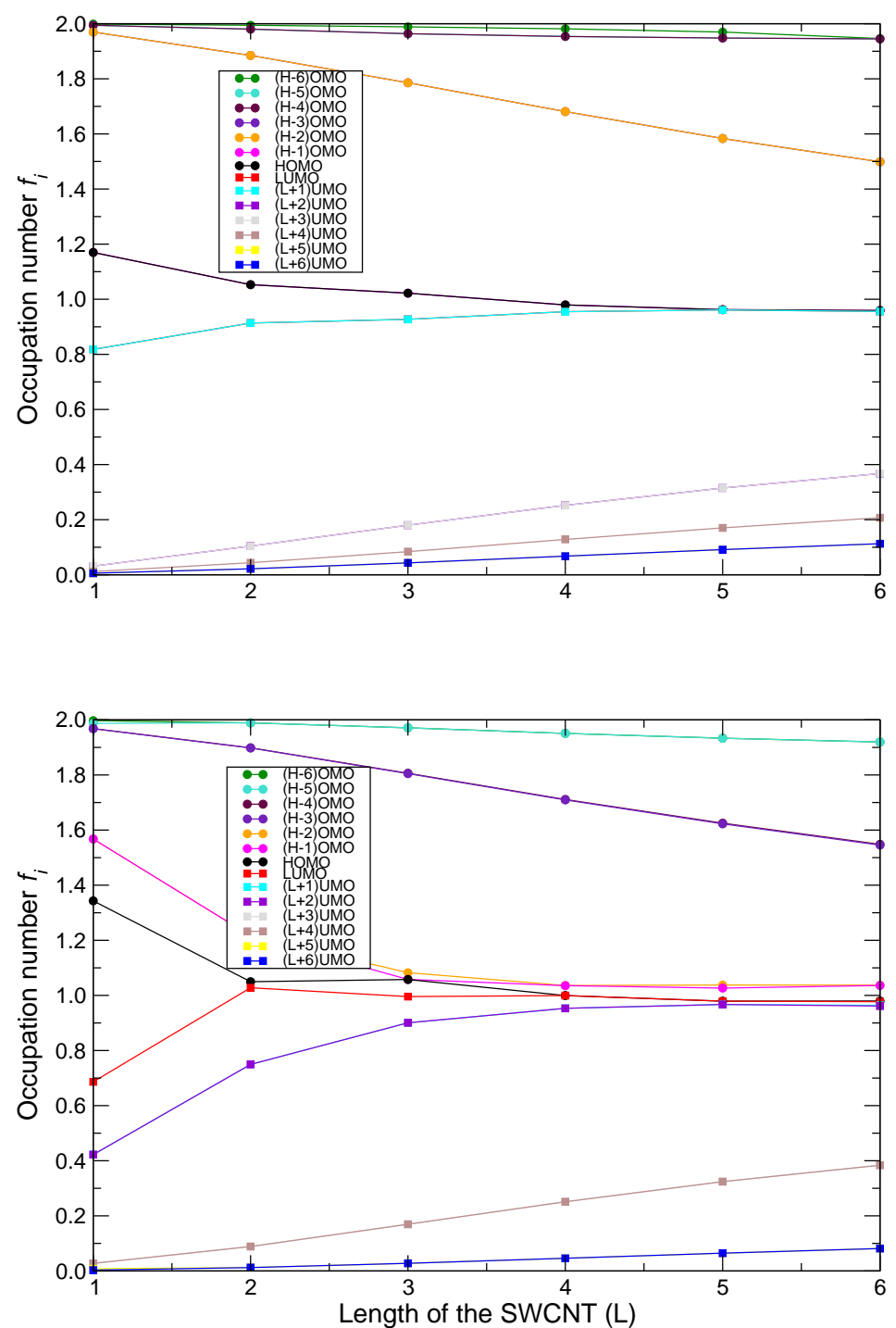

Figure 6: Evolution of $f_{i}$ values for [9]CC (top) and [12]CC (bottom), as obtained from the FT-DFT method, for the (H-6)OMO to $(\mathrm{L}+6) \mathrm{UMO}$ set of orbitals. 

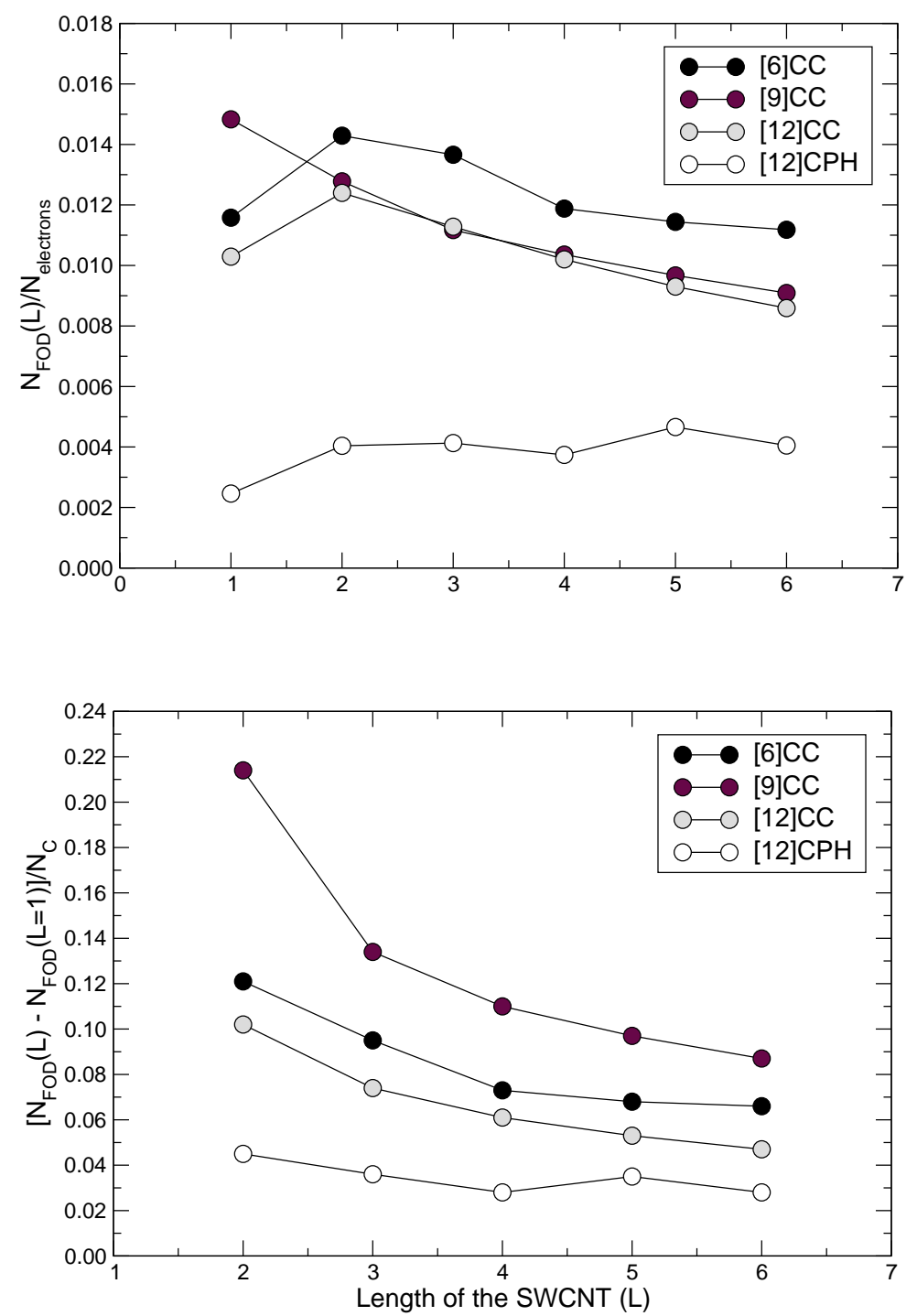

Figure 7: Evolution of $\mathrm{N}_{\mathrm{FOD}}$ values as obtained from the FT-DFT method, renormalized by the number of electrons (top) and by attenuating edge effects (bottom) for each nanotube size $(L=1-6)$. 


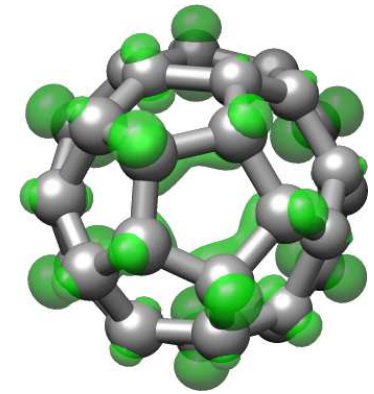

$\mathrm{N}_{\mathrm{FOD}}=1.76$

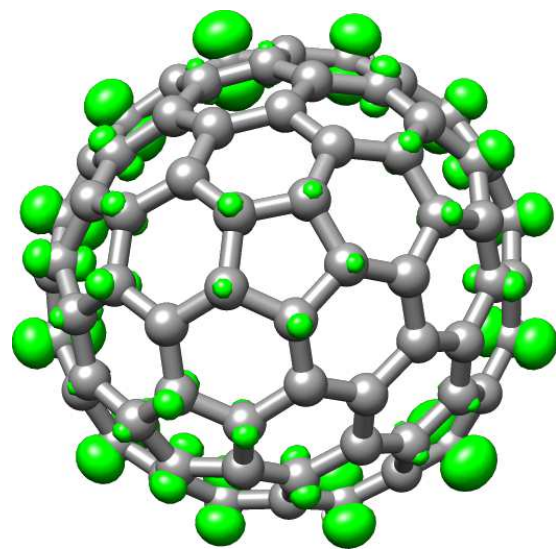

$\mathrm{N}_{\mathrm{FOD}}=3.92$

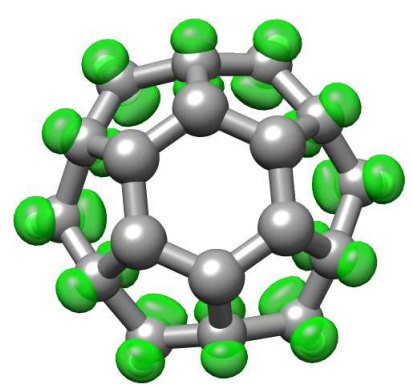

$\mathrm{N}_{\mathrm{FOD}}=1.36$

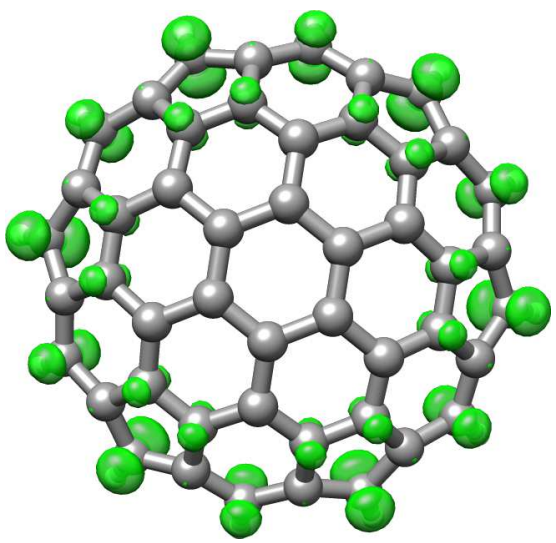

$\mathrm{N}_{\mathrm{FOD}}=3.18$

Figure 8: Chemical structures and plots $\left(\sigma=0.005 \mathrm{e} / \mathrm{bohr}^{3}\right)$ of the FOD density obtained from the FT-DFT method for the caps with pentagonal (left) and hexagonal (right) base, for both [6]CC (top) and [12]CC (bottom) systems. 


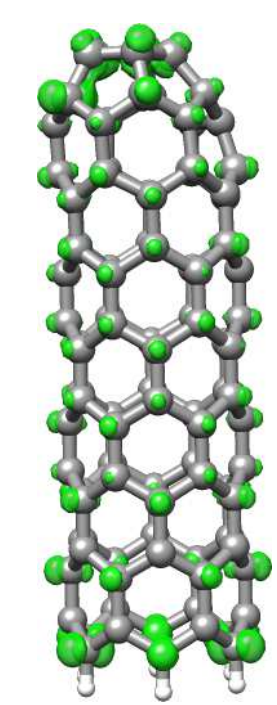

$\mathrm{N}_{\mathrm{FOD}}=6.37$

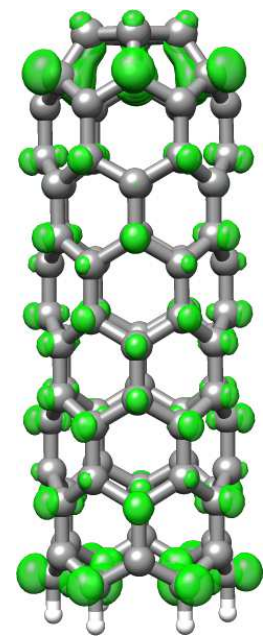

$\mathrm{N}_{\mathrm{FOD}}=5.98$

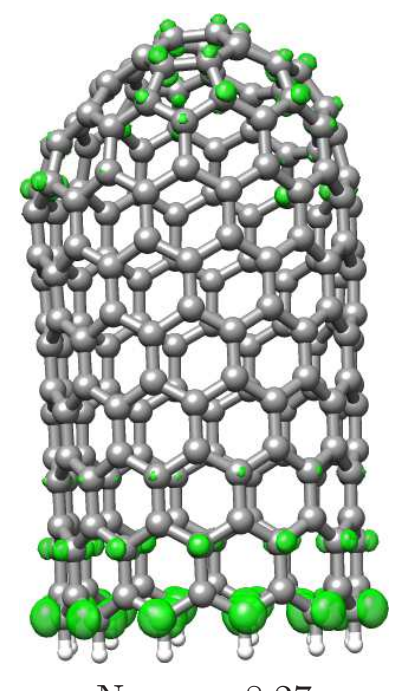

$\mathrm{N}_{\mathrm{FOD}}=8.27$

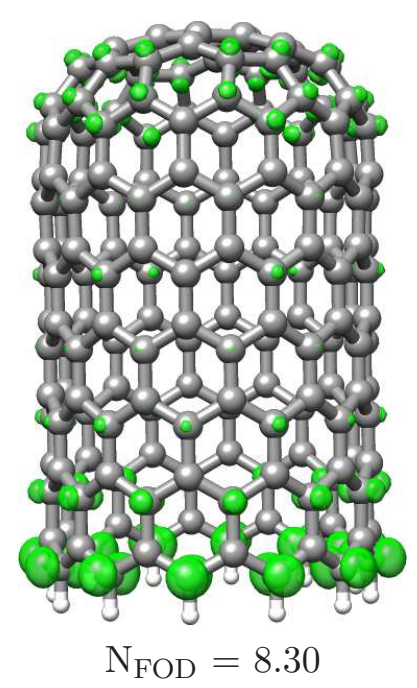

Figure 9: Chemical structures and plots $\left(\sigma=0.005 \mathrm{e} / \mathrm{bohr}^{3}\right)$ of the FOD density obtained from the FT-DFT method for one-side end-capped SWCNT with pentagonal (left) and hexagonal (right) caps base, for both [6]CC (top) and [12]CC (bottom) systems. 


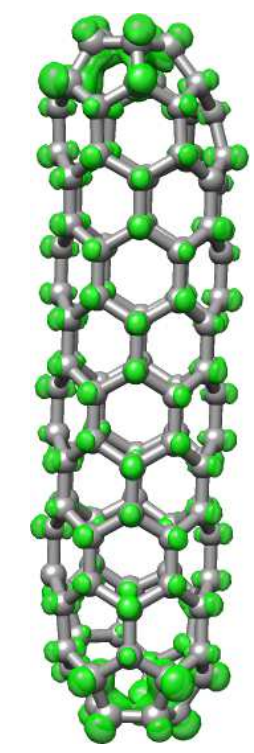

$\mathrm{N}_{\mathrm{FOD}}=7.24$

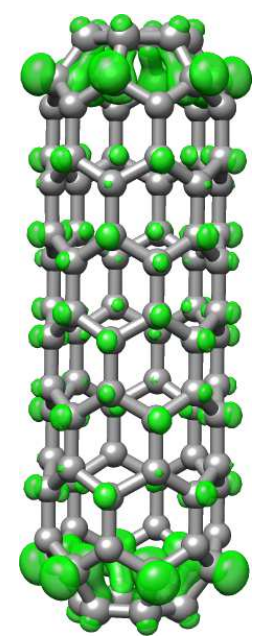

$\mathrm{N}_{\mathrm{FOD}}=6.36$

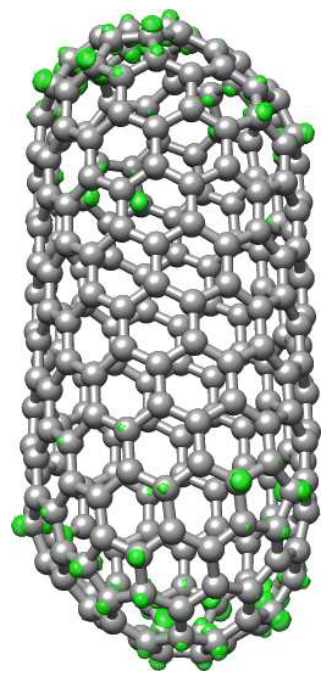

$\mathrm{N}_{\mathrm{FOD}}=8.60$

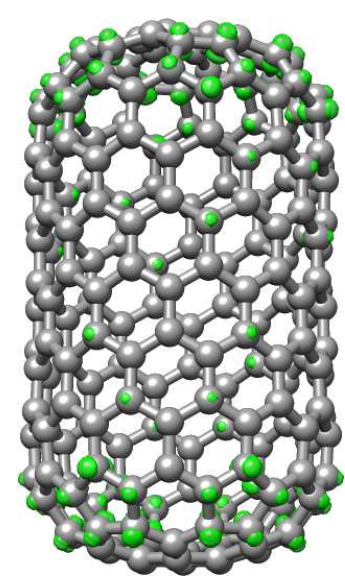

$\mathrm{N}_{\mathrm{FOD}}=7.88$

Figure 10: Chemical structures and plots $\left(\sigma=0.005 \mathrm{e} / \mathrm{bohr}^{3}\right)$ of the FOD density obtained from the FT-DFT method for two-sides end-capped SWCNT with pentagonal (left) and hexagonal (right) caps base, for both [6]CC (top) and [12]CC (bottom) systems. 